\title{
Experimental observation of constructive superposition of wakefields generated by electron bunches in a dielectric-lined waveguide
}

\author{
S. V. Shchelkunov, ${ }^{1}$ T. C. Marshall, ${ }^{1}$ J. L. Hirshfield, ${ }^{2,4}$ M. A. Babzien, ${ }^{3}$ and M. A. LaPointe ${ }^{2}$ \\ ${ }^{1}$ Department of Applied Physics, Columbia University, New York City, New York 10027, USA \\ ${ }^{2}$ Department of Physics, Yale University, New Haven, Connecticut 06520-8120, USA \\ ${ }^{3}$ Brookhaven National Laboratory, New York 11973, USA \\ ${ }^{4}$ Omega-P, Inc., New Haven, Connecticut 06520, USA \\ (Received 2 June 2005; published 12 January 2006)
}

\begin{abstract}
We report results from an experiment that demonstrates the successful superposition of wakefields excited by $50 \mathrm{MeV}$ bunches which travel $\sim 50 \mathrm{~cm}$ along the axis of a cylindrical waveguide which is lined with alumina. The bunches are prepared by splitting a single laser pulse prior to focusing it onto the cathode of an rf gun into two pulses and inserting an optical delay in the path of one of them. Wakefields from two short (5-6 psec) $0.15-0.35 \mathrm{nC}$ bunches are superimposed and the energy loss of each bunch is measured as the separation between the bunches is varied so as to encompass approximately one wakefield period $(\sim 21 \mathrm{~cm})$. A spectrum of $\sim 40 \mathrm{TM}_{0 m}$ eigenmodes is excited by the bunch. A substantial retarding wakefield $(2.65 \mathrm{MV} / \mathrm{m} \cdot \mathrm{nC}$ for just the first bunch) is developed because of the short bunch length and the narrow vacuum channel diameter $(3 \mathrm{~mm})$ through which they move. The energy loss of the second bunch exhibits a narrow peak when the bunch spacing is varied by only $4 \mathrm{~mm}(13.5 \mathrm{psec})$. This experiment is compared with a related experiment reported by a group at the Argonne National Laboratory where the bunch spacing was not varied and a much weaker retarding wakefield $(\sim 0.1 \mathrm{MV} / \mathrm{m} \cdot \mathrm{nC}$ for the first bunch) comprising only about 10 eigenmodes was excited by a train of long $(\sim 9 \mathrm{~mm})$ bunches.
\end{abstract}

DOI: 10.1103/PhysRevSTAB.9.011301

PACS numbers: 41.75.Lx, 29.17.+w, 41.60.Bq

\section{INTRODUCTION}

Wakefield accelerators are generally attractive because no external source of energy is used in the structure itself, and all the accelerating fields are generated from only bunches themselves. The source of power required for acceleration of the drive bunches can be provided by an efficient conventional $\mathrm{rf}$ linac. High acceleration fields are believed to be possible in the dielectric because it is exposed to intense fields for only a very short time. The breakdown limit of the wakefield structure is not determined by the slow filling time of the structure by electromagnetic energy, but rather by the much shorter time of the passing field pulses set up by the short bunches which build up the wakefields.

In 1999, some new refinements were proposed to boost the acceleration gradient in dielectric-lined waveguides [1]. Comparatively short drive bunches were suggested, so that excitation of a large number of high-amplitude $\mathrm{TM}_{0 m}$ eigenmodes would form a high-amplitude wakefield. The waveguide design [2] would be chosen so that the wakefield is nearly periodic, with the same period as that of the train of drive bunches. If several drive bunches can be used to excite a dielectric wakefield apparatus (DWA), the $E_{z}$ field at the test bunch (a bunch that undergoes acceleration) location will have a much higher amplitude than that which would be set up by only one bunch. The use of a train of colinear bunches is helpful when it is not possible to form a bunch which has the aggregate charge of the bunch train. In this acceleration scheme, all drive bunches must radiate coherently so that every con- secutive drive bunch enhances the wakefield produced by the drive bunches which have preceded it; in this case a constructive superposition of wakefields generated by the drive bunches occurs.

The interaction between the wakefield and electron bunches in cylindrical dielectric-lined waveguides has been theoretically analyzed in recent years [1,3-7]. However, there has been only one experimental study of constructive wakefield superposition, confirmation of which is crucial to prove the feasibility of dielectric wakefield acceleration. Constructive superposition of wakefields produced by several bunches has been observed only by a group at Argonne National Laboratory [8]. In their case, the wakefield is excited by relatively long bunches (rms length $\sim 9 \mathrm{~mm}$ ) in a $\varnothing 10 \mathrm{~mm}$ channel, consists of $\sim 10$ eigenmodes, and has an $E_{z}$ field with a broad footprint $\left(2 \sigma_{\text {wake }} \sim 22 \mathrm{~mm}\right)$ but a low amplitude (decelerating wakefield after one bunch $\sim 32 \mathrm{kV} / \mathrm{m} \cdot \mathrm{nC}$ ). In contrast, the experiment conducted at the Accelerator Test Facility (ATF) on which we report here generates a wakefield having 40 modes and a decelerating field after one bunch $\sim 980 \mathrm{kV} / \mathrm{m} \cdot \mathrm{nC}$. The high axial $E_{z}$ field with a narrow footprint $\left(2 \sigma_{\text {wake }} \sim 4 \mathrm{~mm}\right)$ is achieved because of narrow (rms length $\sim 1.8 \mathrm{~mm}$ ) bunches (hence permitting the excitation of much more modes) and a narrow channel (Ø3 mm).

Our observation technique is different from that used in the Argonne experiment, where the measured energy spectrum of drive bunches was compared with a computed energy spectrum while the bunch spacing was fixed and 
equal to the wakefield period. In our case, the bunch spacing is changed, and the difference in energy losses between the second and first bunches is observed. This allows one to compare theory and experiment when the bunch spacing differs from the wakefield period, and to observe thereby the spatial profile of the wakefields on the axis following the bunches.

We use a cylindrical dielectric structure to test these physical principles. However, it has been pointed out that a rectangular structure made from slabs of dielectric is superior to the cylinder in that the mode dispersion is smaller and that the stability of the bunches is better; thus a longer train of bunches may be used to construct a larger wakefield than in the example of the cylinder. Rectangular structures for wakefield acceleration have been recently analyzed [9-11].

\section{MONITORING THE ENERGY LOSSES OF CONSECUTIVE BUNCHES AS A TOOL FOR OBSERVATION OF CONSTRUCTIVE WAKEFIELD SUPERPOSITION}

First, a brief summary is presented of analysis of the interaction between electron bunches and wakefields in a cylindrical dielectric-lined waveguide. The theory has been thoroughly developed in recent years [3-7]. The wakefield is assumed to be excited by passage of a train of $N$ drive bunches which moves with the velocity $\nu=c \beta$ along the axis ( $z$ direction) in a dielectric-lined waveguide. There is vacuum in the region $r<A$, a dielectric material with constant dielectric coefficient $\varepsilon$ everywhere between $A<r<R$, and a metal conducting pipe at $r \geq R$, where $A$ is the inner radius and $R$ is the outer radius. In cylindrical geometry, the wakefield can be described as a superposition of orthonormal wave functions which separate into TE and TM classes for axisymmetric excitation, and into hybrid modes with mixed polarization otherwise [3]. The contributions from transition radiation localized near axial boundaries of the dielectric insert are neglected (an unbounded structure approximation) [12].

Assuming that all bunches have the same longitudinal shape, but different charges, the power (computed through the modified Poynting vector [3]) which is radiated by $N$ bunches is

$$
P_{N}=\frac{c \beta}{4 \pi \varepsilon_{0} A^{2}} \sum_{m} \Omega_{m} \sum_{i, j}^{N, N} Q_{i} Q_{j} \cos \left(\frac{\omega_{m} S_{i j}}{c \beta}\right)
$$

where $S_{i j}=z_{i}-z_{j}$ is the spacing between the $i$ th and $j$ th bunches. The coefficient $\Omega_{m}=\alpha_{m}^{-1}\left[\left(\Gamma_{C}^{m}\right)^{2}+\left(\Gamma_{S}^{m}\right)^{2}\right]=$ $\Omega\left(\omega_{m}, \Delta z\right)$ depends weakly on the bunch shape $[13,14]$ but is determined by and sensitive to the rms bunch length. Using this fact, one can assume that the bunch longitudinal shape is always Gaussian; then $\Gamma_{C}^{m}=$ $\exp \left(-\omega_{m}^{2} \Delta z^{2} / 8 c^{2} \beta^{2}\right)$ and $\Gamma_{S}^{m}=0$.
In Eq. (1), $Q_{i}$ is the charge of the $i$ th bunch; $z_{i}$ is the position of the $i$ th bunch on the $z$ axis; the rms length is defined as $\Delta z=2 \sqrt{\overline{z^{2}}-\bar{z}^{2}} ; \alpha_{m}$ is the normalizing constant [1]; and $\Gamma_{C}^{m, i}=\int_{-\infty}^{\infty} d z_{0}^{\prime} F_{i}\left(z_{0}^{\prime}\right) \theta_{h v}\left(z_{0}^{\prime}-z_{0}\right) \times$ $\cos \left(\frac{\omega_{m}}{c \beta} z_{0}^{\prime}\right)$, and $\Gamma_{S}^{m, i}=\int_{-\infty}^{\infty} d z_{0}^{\prime} F_{i}\left(z_{0}^{\prime}\right) \theta_{h v}\left(z_{0}^{\prime}-z_{0}\right) \times$ $\sin \left(\frac{\omega_{m}}{c \beta} z_{0}^{\prime}\right)$, where function $F_{i}$ describes the longitudinal shape of the $i$ th bunch, and $\theta_{h v}$ is the Heaviside function.

Equation (1) clearly demonstrates that the radiation is emitted in a coherent fashion.

Every drive bunch loses a very specific amount of energy per unit of length

$$
W(N)=\left(P_{N}-P_{N-1}\right) / c \beta Q_{N} .
$$

For the first bunch $(N=1)$, the energy loss is

$$
W(1) / Q_{1}=\left(4 \pi \varepsilon_{0} A^{2}\right)^{-1} \sum_{m} \Omega\left(\omega_{m}, \Delta z\right) .
$$

Since $\Omega\left(\omega_{m}, \Delta z\right)$ weakly depends on the bunch shape, the first bunch energy loss is negligibly affected by the bunch shape. The bunch energy loss is directly proportional to the bunch charge. For the second bunch $(N=2)$ one obtains

$$
\begin{aligned}
\frac{W(2)}{Q_{2}}= & \frac{W(1)}{Q_{1}} \times\left(1+2 \frac{Q_{1}}{Q_{2}}\right. \\
& \left.\times \frac{\sum_{m} \Omega\left(\omega_{m}, \Delta z\right) \cos \left(\omega_{m} S_{12} / c \beta\right)}{\sum_{m} \Omega\left(\omega_{m}, \Delta z\right)}\right),
\end{aligned}
$$

where $W(1) / Q_{1}$ is given above, and $S_{12}$ is the distance between bunches (bunch spacing), and we assume that both bunches have the same rms length.

The behavior of $W(2) / Q_{2}$ vs the bunch spacing is shown in Fig. 1. It shows an enhancement in magnitude when the bunch spacing is in the vicinity of $J L$ (where $L$ is the wakefield period and $J$ is any integer), which can be used as an indicator of the constructive wakefield superposition happening when $S_{12} \rightarrow L$. We should point out that the full width of this peaking curve is the same as the wakefield full footprint $2 \sigma_{\text {wake }}$ [14]. In general, if every subsequent bunch enhances the wakefield produced by the first bunch (constructive wakefield superposition), the energy losses are always such that

$$
\begin{aligned}
& W(N) / Q_{N}>W(N-1) / Q_{N-1}>\ldots>W(2) / Q_{2} \\
& \quad>W(1) / Q_{1},
\end{aligned}
$$

where it is assumed that all bunches have the same rms length.

In an experiment, it is convenient to use the first bunch as a reference bunch, because its energy loss does not depend on the bunch spacing, and to measure the difference $W(N)-W(1)$ vs the bunch spacing. Because usually $Q_{N} \approx$ $\ldots \approx Q_{2} \approx Q_{1}$ and $\Delta z_{N} \approx \ldots \approx \Delta z_{2} \approx \Delta z_{1}$, this difference must be positive whenever the constructive superpo- 


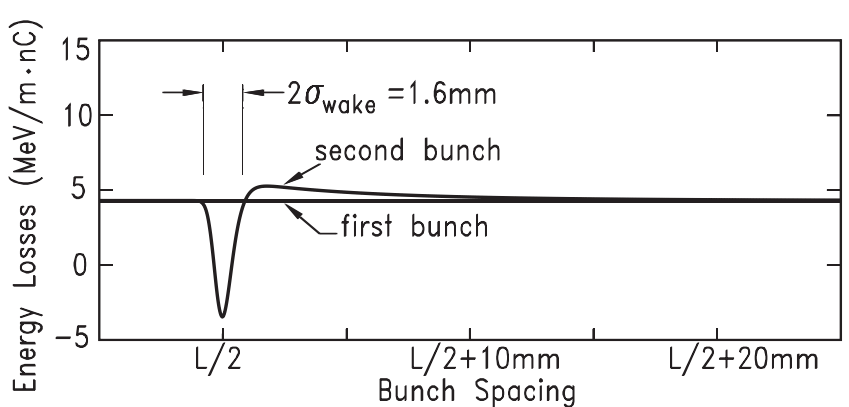

a)

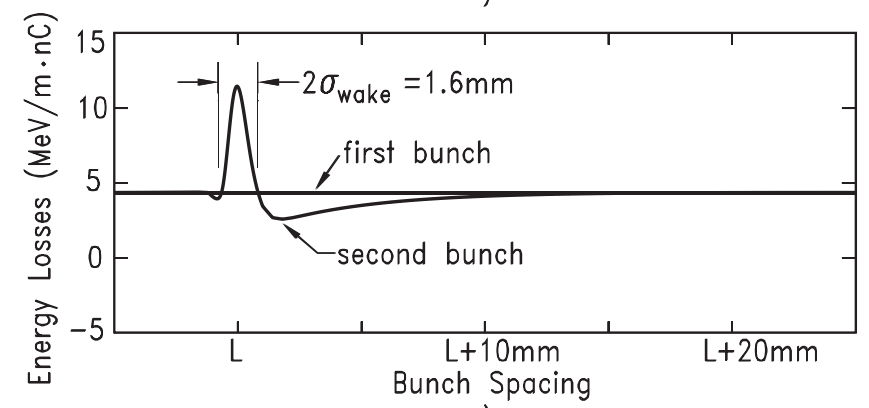

b)

FIG. 1. The behavior of $W(1) / Q_{1}$ and $W(2) / Q_{2}$ vs the spacing between two bunches. $W(1) / Q_{1}$ is determined by Eq. (3) and does not depend on the bunch spacing (marked by "first bunch"). $W(2) / Q_{2}$ is determined by Eq. (4) and changes with the bunch spacing (marked by "second bunch"). If the bunch spacing, $S_{12}$, is close to $J L / 2$ the value of $W(2) / Q_{2}$ is significantly different from $W(1) / Q_{1}$. In particular, when $S_{12} \rightarrow$ $L / 2$ [see (a)] the second bunch is accelerated; but when $S_{12} \rightarrow L$ [see (b)] the second bunch is decelerated, and consequently, enhances the wakefield produced by the first bunch. (Here $2 \sigma_{\text {wake }}=1.6 \mathrm{~mm}$ is the full wakefield footprint, the wakefield period $L=0.21022 \mathrm{~m}, A=1.5 \mathrm{~mm}, R=19.31 \mathrm{~mm}$, and $\varepsilon=9.65)$. sition of fields occurs, and therefore an enhancement of the wakefield must occur whenever the bunch spacing $S_{12}=$ $S_{\text {bunch }}$ is varied in the vicinity of the wakefield period, $L$.

\section{EXPERIMENT}

This experiment has been run on beam line \#2 at ATF. Figure 2 shows the experimental setup. HeNe-laser light is used to establish the apparatus axis. The optical beam is focused in the middle plane of the DWA, and has waist size at the entrance and exit of the DWA $w \approx 2 A / 3$ (where $A=$ $1.5 \mathrm{~mm}$ is the inner radius of the vacuum channel), so that clear transmission occurs only if the light beam propagates along the DWA axis. The electron bunch path is aligned with the HeNe light before the bunch enters the DWA. Numerical simulations show that an electron bunch propagating along the axis of the apparatus is negligibly distorted by the transverse wakefields [14]. Upon leaving the DWA, this bunch will therefore have the same transverse size as a bunch that moves in free space (i.e., the transverse dynamics will be accurately described as if there was no dielectric insert). Diagnostics available at ATF permit measuring the horizontal and vertical emittances [typically, the non-normalized emittance is in the range $(0.85-2.6) \times 10^{-8} \mathrm{mrad}$ at charges $Q \approx 150 \mathrm{pC}-\ln C$ ] and initial Twiss parameters so that the transverse bunch sizes in the free-space approximation can be calculated at any point along beam line \#2. The measurement of transverse size after the bunch leaves the DWA demonstrates excellent agreement between the measured and expected values $\left(\sigma_{x, y} \approx 250-300 \mu \mathrm{m}\right)$ as soon as the bunch is aligned, and serves as a criterion to confirm that the bunch deviates negligibly from the apparatus axis.

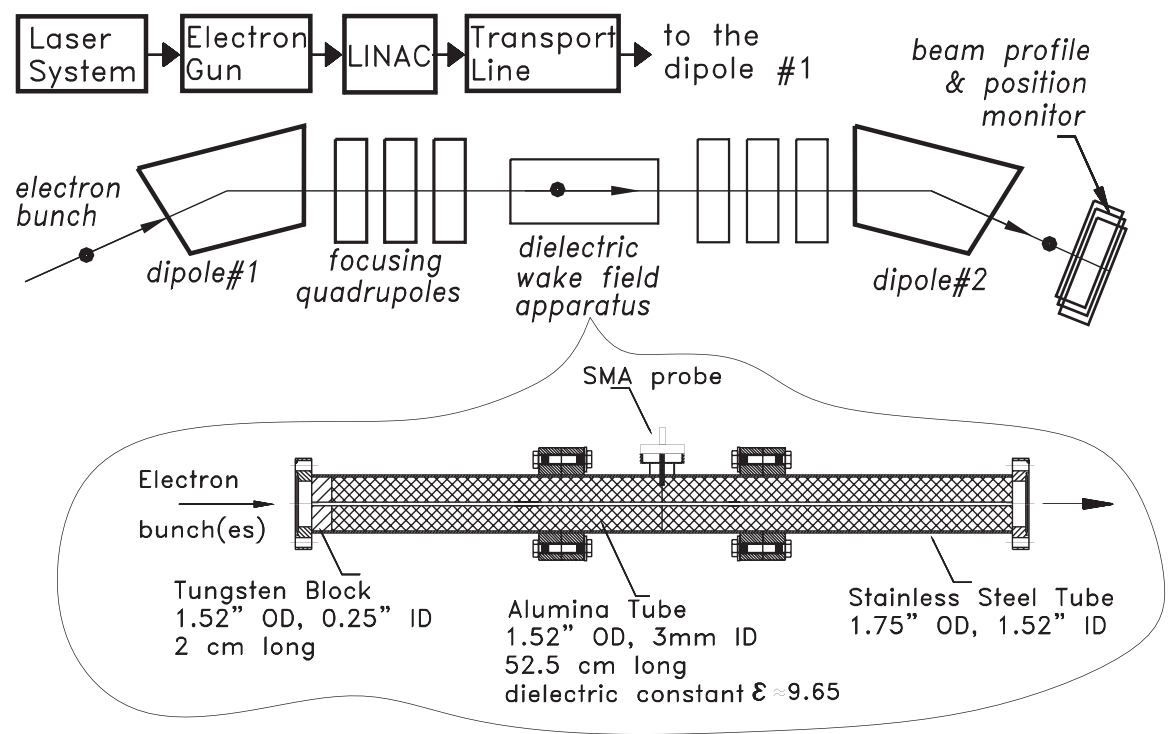

FIG. 2. Schematic of the experimental setup and the dielectric wakefield apparatus (DWA). 
Diagnostic information, such as bunch charge and rms length, is obtained routinely from the permanent ATF facility hardware available to all users. The bunch charge is measured by a Faraday cup. To measure the rms bunch length, a variable phase shift is introduced for the second $\mathrm{rf}$ linac section. A particle located at the head of the bunch thereby gains a different energy than one located at the tail. Thus the bunch profile along the time axis is mapped onto an energy axis. After passing through a dipole (the first dipole in the transport line [not shown in Fig. 2]), the bunch trajectory experiences a transverse shift for every energy shift. Using a slit followed by a monitor, one can scan the charge profile of the bunch as the linac phase is varied (about $0.973 \mathrm{psec} / \mathrm{deg}$ ).

In the experiment, the first bunch is used as a reference bunch. A part of the transport line allows measurement of the initial energy difference between bunches, $\Delta W_{\text {ini }}=$ $E_{\text {ini }}(N)-E_{\text {ini }}(1)$. The beam position monitor installed after the dipole \#2 [see Fig. 2] is used to measure the final energy difference $\Delta W_{\text {fin }}=E_{\text {fin }}(N)-E_{\text {fin }}(1)$. Thus, the difference in energy losses, $W(N)-W(1)=-\Delta W_{\text {fin }}+\Delta W_{\text {ini }}$, caused by interaction with the wakefield in the DWA is determined.

To produce several electron bunches, the single laser light pulse ( $F W H M \approx 7$ psec) delivered to the ATF photocathode gun with the repetition rate $1.5 \mathrm{~Hz}$ is split into several pulses with the separation between them close to $700 \mathrm{psec}(21 \mathrm{~cm})$. The optical stage [Fig. 3] can produce up to four pulses (the separation between the third and fourth ones is close to $350 \mathrm{psec}$ ), and is used to adjust the bunch spacing.

In the experiment described, the wakefield period cannot be adjusted, but there is also a finite dispersion function at the DWA location. Because of the finite dispersion, the bunch spacing cannot differ much from an integer of the period of rf, which drives the electron gun and LINAC, because otherwise the bunches would have an energy difference, and that energy difference would cause them to propagate along different trajectories inside the DWA. As a result, if the first bunch propagates along the DWA axis, the second bunch trajectory would deviate from the apparatus axis. For instance, with the typical dispersion function obtained in the ATF experiment $\left(D_{x} \approx D_{y} \approx\right.$ $0.7 \mathrm{~cm}$ ), the second bunch would deviate from the apparatus axis by less than $\sigma_{x, y}$ (where $\sigma_{x, y} \approx 250 \mu \mathrm{m}$ ) only if the drive bunch spacing $S_{\text {bunch }}$ differs from an even integer of the $\mathrm{rf}$ period $2 \boldsymbol{J} \lambda_{\mathrm{rf}}$ by no more than 7 psec (i.e., $\left|S_{\text {bunch }}-2 \boldsymbol{J} \lambda_{\mathrm{rf}}\right| \leq 7$ psec, where $\boldsymbol{J}$ is any integer and $\lambda_{\mathrm{rf}}$ is the rf period.)

If the wakefield period is different from $2 \boldsymbol{J} \lambda_{\mathrm{rf}}$, only a limited number of drive bunches whose wakefields add up constructively can be transmitted through a DWA. It will be demonstrated later that for this experiment, constructive superposition occurs if $S_{\text {bunch }}=\boldsymbol{J} L=\boldsymbol{J}\left(2 \lambda_{\mathrm{rf}}+3.7 \mathrm{psec}\right)$ [e.g., see Eqs. (5a) and (5b)]. One finds, then, that the total

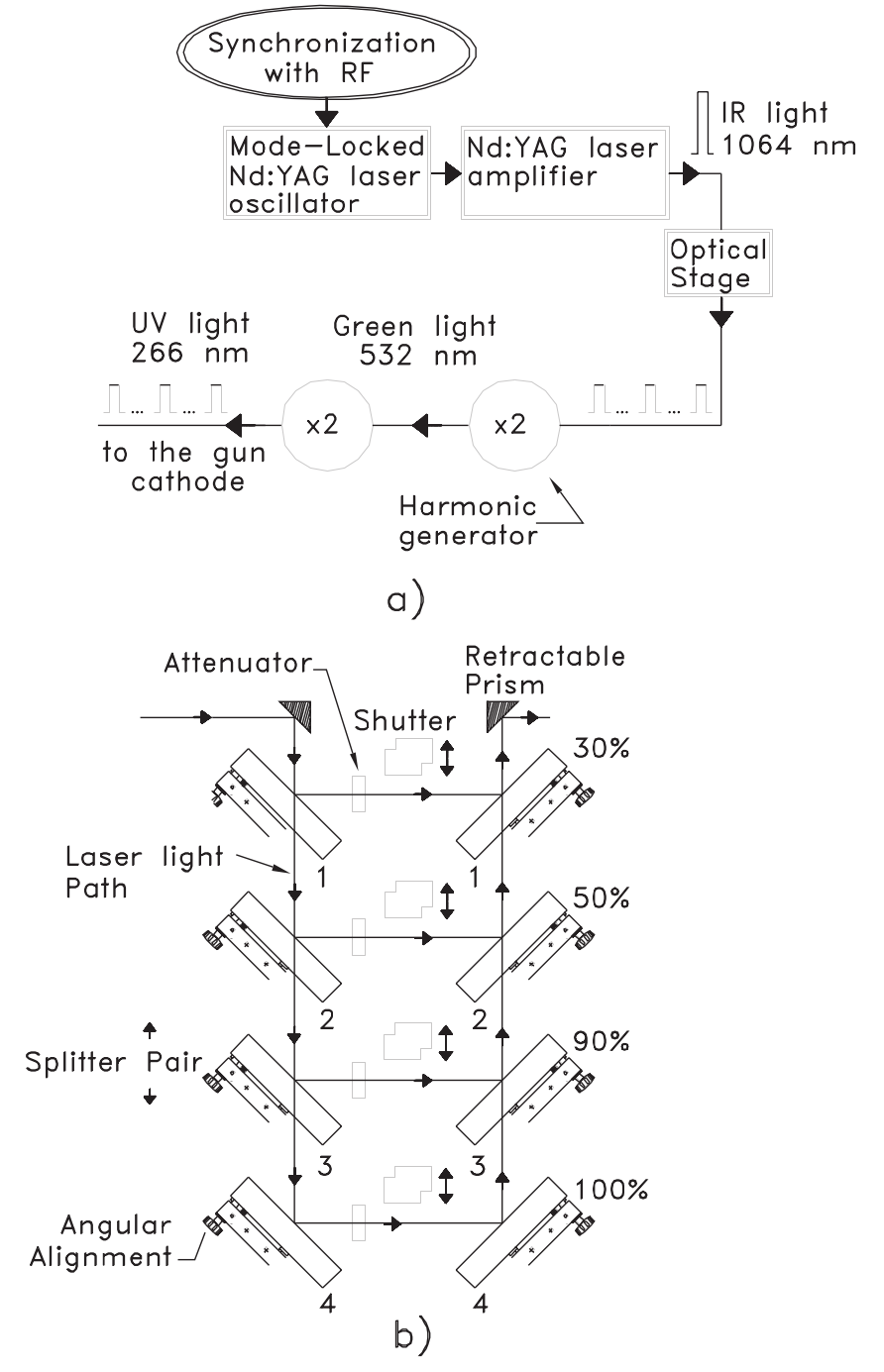

FIG. 3. (a) The ATF laser system schematic with the optical stage, and (b) the optical stage schematic. Percentages refer to the mirror reflectance.

number of drive bunches $N$ cannot exceed $N \leq \boldsymbol{J}+1 \leq$ $\frac{7 \mathrm{psec}}{3.7 \mathrm{psec}}+1 \approx 3$, with acceptance of the $3 \mathrm{rd}$ bunch being marginal. Thus in what follows, we discuss observations that arose when we adjusted the spacing of only two bunches, using the optical delay, to superimpose the wakefields.

The bunch spacing $S_{\text {bunch }}=700.28$ psec $+\Delta S_{\text {bunch }}$ is varied by changing the laser pulse spacing $S_{\text {laser }}=$ $700.28 \mathrm{psec}+\Delta S_{\text {laser }}$. All distances (in units of time) and corresponding measurement/calibration errors (which include the accuracy of establishing the frequency) are measured/evaluated from $700.28 \mathrm{psec}$, which is double the rf period at $2856 \mathrm{MHz}$.

$\Delta S_{\text {laser }}$ is usually changed by \pm several psec and can be directly measured with the optical stage. To relate $\Delta S_{\text {bunch }}$ with $\Delta S_{\text {laser }}$ one constructs the 1D phase-energy space mapping [14-16], and convolves it with the initial 
longitudinal bunch distribution at the gun cathode. The initial bunch charge distribution is determined by the distribution of the laser pulse energy delivered to the cathode. The phase-energy mapping depends upon the gun maximum electric field $E_{\text {gun }}$, the LINAC electric field $E_{\text {linac }}$ that is set to achieve the final bunch energy of $50 \mathrm{MeV}$, the gun operational phase $\varphi_{\text {gun }}$, and the corresponding LINAC phase $\varphi_{\text {linac }}$ which minimizes the energy spread. The mapping should be applied to bunches with a relatively low charge [e.g., see Figs. 5(a) and 5(b)]. The criterion of validity of the mapping is that the calculated rms bunch length (and/or FWHM) is, within the measurement accuracy, the same as the measured rms length. The energy loss data were gathered for two cases, for which the relationship between $\Delta S_{\text {bunch }}$ and $\Delta S_{\text {laser }}$ is shown in Figs. 4(a) and 4(b). In Fig. 4(a) (first case), with $\Delta S_{\text {laser }}$ changing from -1.39 to $+5.07 \mathrm{psec}, \Delta S_{\text {bunch }}$ changes from -1.24 to +4.88 psec. In Fig. 4(b) (second case), with $\Delta S_{\text {laser }}$ changing from -4.87 to $+4.47 \mathrm{psec}, \Delta S_{\text {bunch }}$ changes from -5.75 to +6.62 psec.

Enough data have been collected to demonstrate the enhanced wakefield amplitude behavior of $W(2)-W(1)$ vs the bunch spacing for both cases. The bunch charges (measured in the experiment) change from one data point

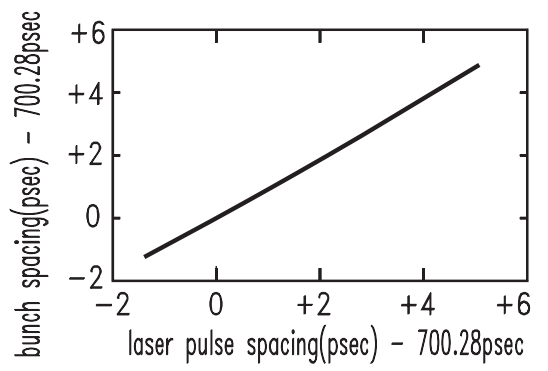

$$
\begin{aligned}
& \varphi_{\text {gun }} \approx 59.4^{0} \\
& \varphi_{\text {linac }} \approx-17.9^{\circ} \\
& \mathrm{E}_{\text {gun }} \approx 100 \mathrm{MV} / \mathrm{m} \\
& \mathrm{E}_{\text {linac }} \approx 7.6 \mathrm{MV} / \mathrm{m}
\end{aligned}
$$

a) first case

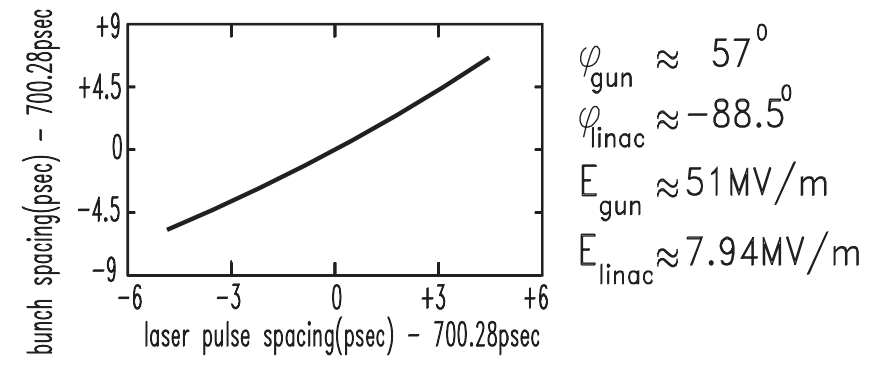

b) second case

FIG. 4. Bunch spacing vs the laser spacing for (a) $\varphi_{\text {gun }} \gg$ $59.4^{\circ}$ (measured), $\varphi_{\text {linac }} \approx-17.9^{\circ}$ (adjusted to minimize the energy spread), $E_{\text {gun }} \approx 100 \mathrm{MV} / \mathrm{m}$ (measured), and $E_{\text {linac }} \approx$ $7.6 \mathrm{MV} / \mathrm{m}$ (adjusted to achieve the final bunch energy of $50 \mathrm{MeV}$ ). (The corresponding difference in bunch energy losses, $W(2)-W(1)$, is shown in Fig. 6(a), and the bunch charges are shown in Fig. 5(a)]; (b) $\varphi_{\text {gun }} \gg 57^{\circ}, \varphi_{\text {linac }} \approx-88.5^{\circ}, E_{\text {gun }} \approx$ $51 \mathrm{MV} / \mathrm{m}$, and $E_{\text {linac }} \approx 7.94 \mathrm{MV} / \mathrm{m}$. (The corresponding difference in bunch energy losses, $W(2)-W(1)$, is shown in Fig. 6(b) [the bunch charges are shown in Fig. 5(b)]). to another as shown in Fig. 5(a) (first case) and Fig. 5(b) (second case).

Figure 6(a) (first case) presents the measured difference in energy losses (marked by bars) between the second and first bunches, $W(2)-W(1)$, versus $\Delta S_{\text {bunch }}$ when both bunches have the same rms length $\Delta z=2 \sqrt{\overline{z^{2}}-\bar{z}^{2}}=$ $5.4 \pm 0.2 \mathrm{psec}$. The solid curve connects the points which give the best theoretical fit which happens if the wakefield period is taken to be

$$
L=700.28 \mathrm{psec}+\Delta L=700.28 \mathrm{psec}+3.6 \mathrm{psec},
$$

where $\Delta L$ is determined with an accuracy $\pm 10.5 \%$ (i.e., the wakefield period is determined with an accuracy $\pm 0.38 \mathrm{psec} \approx \pm 115 \mu \mathrm{m}$ ). The solid curve has a piecewise character because the bunch charges change from one experimental point to another [see Fig. 5(a)].
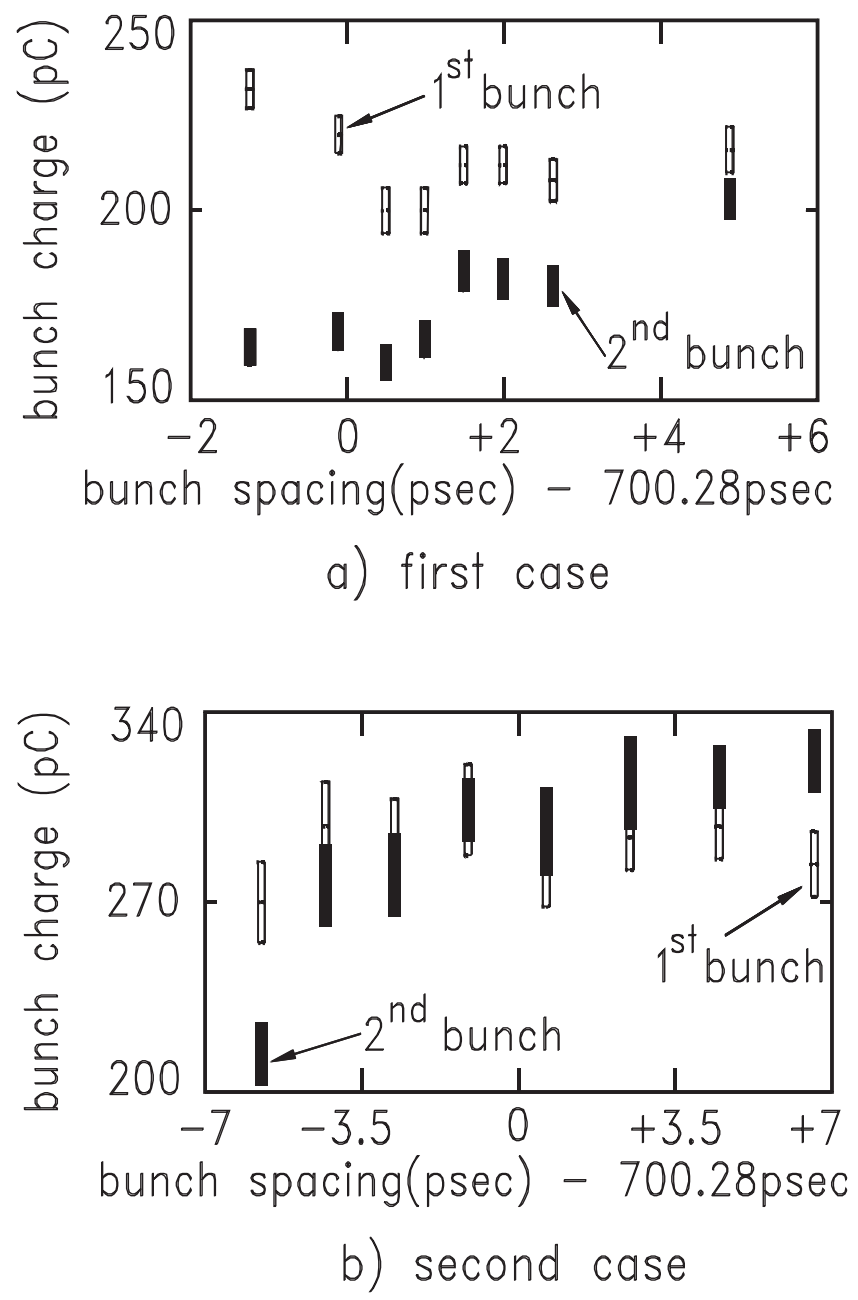

FIG. 5. Charges of the second and first bunches (the accuracy $\approx \pm 3 \%$ ) for which the difference in bunch energy losses, $W(2)-W(1)$, is shown in Fig. 6(a); (b) Charges of the second and first bunches (the accuracy $\approx \pm 5.5 \%$ ) for which the difference in bunch energy losses, $W(2)-W(1)$, is shown in Fig. 6(b). 

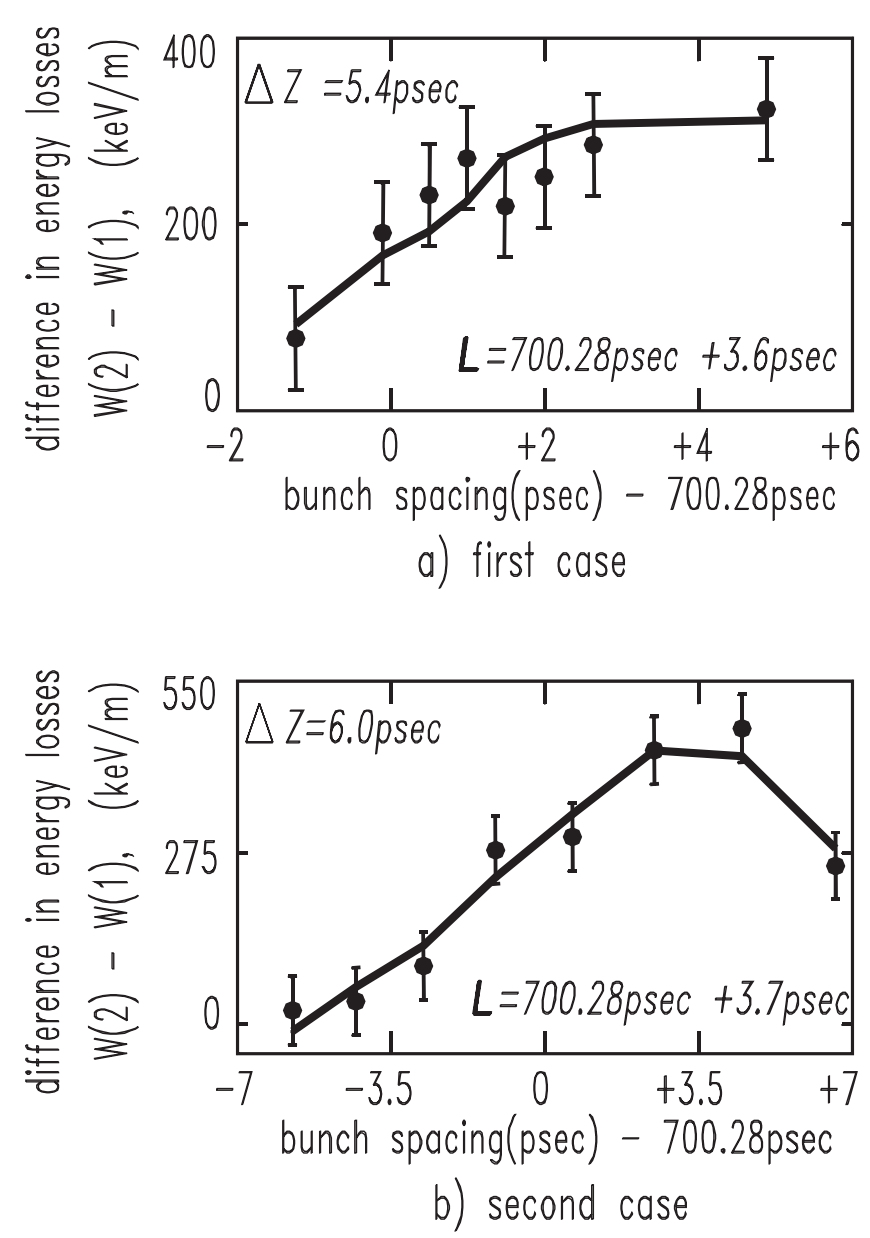

FIG. 6. Measured difference in energy losses (marked by bars; normalized per $1 \mathrm{~m}$ ) between the second and first bunches, $W(2)-W(1)$, vs the bunch spacing: (a) both bunches have the same rms length $\Delta z=5.4 \pm 0.2$ psec. The bunch charges are shown in Fig. 5(a). The solid line represents the best theoretical fit which occurs if the wakefield period is assumed to be $L=$ $700.28 \mathrm{psec}+3.6 \mathrm{psec}$ (or, equivalently, $L=20.994 \mathrm{~cm}+$ $1080 \mu \mathrm{m}$ ); (b) both bunches have the same rms length $\Delta z=$ $6.0 \pm 0.43$ psec. The bunch charges are shown in Fig. 5(b). The solid line represents the best theoretical fit which occurs if the wakefield period is assumed to be $L=700.28 \mathrm{psec}+3.7 \mathrm{psec}$ (or, equivalently, $L=20.994 \mathrm{~cm}+1110 \mu \mathrm{m}$ ).

Figure 6(b) presents the second example. The measured difference $W(2)-W(1)$ vs $\Delta S_{\text {bunch }}$ is given for bunches that have $\Delta z=6.0 \pm 0.43$ psec. Again, the solid curve connects the points which give the best theoretical fit which happens if the wakefield period is taken to be

$$
L=700.28 \mathrm{psec}+\Delta L=700.28 \mathrm{psec}+3.7 \mathrm{psec},
$$

where $\Delta L$ is determined with an accuracy $\pm 10.2 \%$ (the wakefield period is determined with an accuracy $\pm 0.38 \mathrm{psec} \approx \pm 115 \mu \mathrm{m}$ ).

Equation (5a) agrees with Eq. (5b) within the measurement accuracy.

\section{SUMMARY AND DISCUSSION}

The data presented in Figs. 6(a) and 6(b) confirm expectations, and thus, demonstrate that constructive superposition of wakefields occurs as expected (taking the dielectric coefficient to be independent of frequency [17]). From the frequency measurement $[2,18]$, one finds that the wakefield period for the DWA at ATF is $L=700.28 \mathrm{psec}+\Delta L=$ $700.28 \mathrm{psec}+4.08 \mathrm{psec}$, where $\Delta L$ is determined with an accuracy $\pm 10 \%$. This agrees with Eqs. (5a) and (5b) within the measurement accuracy. The frequency measurements and Eqs. (5a) and (5b) establish that the wakefield period is $L=700.28 \mathrm{psec}+\Delta L=700.28 \mathrm{psec}+3.8 \mathrm{psec} \quad$ (with an accuracy of $\pm 0.56 \mathrm{psec} \approx \pm 170 \mu \mathrm{m}$.) Numerical simulations show [14] that to achieve constructive superposition of wakefields in a DWA similar to the one at ATF, the wakefield period must be maintained with an accuracy better than $\pm 0.7 \mathrm{psec} \approx \pm 200 \mu \mathrm{m}$. Thus, the wakefield period is established with sufficient accuracy.

It was described in the previous section how the lack of adjustability of the wakefield period together with the finite dispersion at the DWA location limits the number of bunches whose wakefields add up constructively. One way to overcome this limitation is to further reduce the dispersion function at the DWA location. Regarding the ATF facility, the best DWA location is in the "H line" where the dispersion function is about 10 times or more smaller than in the second experimental line. However, a more comprehensive approach to overcome the limitation on the number of drive bunches is to maintain the bunch spacing equal to an integer of the rf period, but adjust the wakefield period, $L$, so that $L=J \lambda_{\mathrm{rf}}$. Then, the number of drive bunches $N$ can be large. Finding a reliable and straightforward way to tune the wakefield period in a DWA is one of the challenges which must be overcome before the practical realization of the DWA can occur.

Constructive superposition of wakefields produced by several bunches also has been observed by a group at Argonne National Laboratory [8]. In their experiment, the wakefield is excited in a channel with a large radius by long bunches (see Table I) and consists of $\sim 10$ eigenmodes. This yields a lower peak value of the $E_{z}$ field. In contrast, in the experiment conducted at ATF the wakefield having $\sim 40$ modes is excited in a channel with a smaller radius by much shorter bunches. The advantage is that the $E_{z}$ field has a high peak value: for just the first bunch, we observe a decelerating wakefield $\sim 980 \mathrm{kV} / \mathrm{m} \cdot \mathrm{nC}$, whereas Argonne has reported $\sim 32 \mathrm{kV} / \mathrm{m} \cdot \mathrm{nC}$. Thus, in our experiment, electron bunches excite $\sim 30$ times more energetic wakefields [see Table I]. The increase in the wakefield is accounted for in part by the good emittance of the bunch (which permits a smaller diameter vacuum channel) and its shorter length, whereby more modes are excited.

Our scanning technique is different from that of the Argonne group. In their case, the measured energy spec- 
TABLE I. Comparison of the experiments.

\begin{tabular}{|c|c|c|c|c|c|c|}
\hline & $\varepsilon$ & $\begin{array}{l}\text { Inner } \\
\text { radius } \\
(\mathrm{mm})\end{array}$ & $\begin{array}{l}\text { Drive bunch } \\
\text { rms length, } \\
2 \sqrt{\overline{z^{2}}-\bar{z}^{2}} \\
(\mathrm{psec}) /(\mathrm{mm})\end{array}$ & $\begin{array}{c}\text { Number } \\
\text { of } \\
\text { excited } \\
\text { modes }\end{array}$ & $\begin{array}{l}\text { Wakefield full } \\
\text { footprint, } \\
2 \sigma_{\text {wake }} \\
(\mathrm{psec}) /(\mathrm{mm})\end{array}$ & $\begin{array}{c}\text { Peak } \\
\text { accelerating } \\
\text { field after } \\
\text { one bunch }{ }^{\mathrm{c}} \\
(\mathrm{MV} / \mathrm{m} \cdot \mathrm{nC})\end{array}$ \\
\hline Argonne & $38.1^{\mathrm{a}}$ & 5 & $\frac{30}{9}$ & $\sim 10$ & $\frac{74}{22}$ & 0.09 \\
\hline This experiment & $9.65^{\mathrm{b}}$ & 1.5 & $\frac{6}{1.8}$ & $\sim 40$ & $\frac{13.5}{4}$ & 2.65 \\
\hline
\end{tabular}

${ }^{\mathrm{a}}$ Material $\mathrm{CaTiO}_{3}-\mathrm{LaAlO}_{3}$.

${ }^{\mathrm{b}}$ Material $\mathrm{Al}_{2} \mathrm{O}_{3}$.

${ }^{\mathrm{c}}$ The theoretically predicted accelerating gradient for a short $(\Delta z \rightarrow 0)$ test bunch, after the decelerating wakefield acting on the first drive bunch have been measured $(\sim 32 \mathrm{kV} / \mathrm{m} \cdot \mathrm{nC}$ for Argonne and $980 \mathrm{kV} / \mathrm{m} \cdot \mathrm{nC}$ for this experiment).

trum of the drive bunches was compared with a computed energy spectrum, while the bunch spacing was fixed and equal (within a certain accuracy) to the wakefield period (i.e., $S_{\text {bunch }}=L$ ). In the experiment conducted at ATF, we observe the difference in energy losses vs the bunch spacing with the bunch spacing varying in the vicinity of the wakefield period: this revealed the expected behavior [see, e.g., Fig. 1]. This observational technique has two important advantages: the wakefield period can be established with excellent accuracy; and agreement between theory and experiment can be verified when the bunch spacing is different from the wakefield period (i.e., $S_{\text {bunch }} \neq L$ ).

\section{ACKNOWLEDGMENTS}

The authors acknowledge the help received from the ATF staff: T. Watanabe, V. Yakimenko, I. Ben-Zvi, F. Zhou, and D. Davis. This work was supported by the Department of Energy, Division of High Energy Physics.

[1] T.C. Marshall, T-B. Zang, and J.L. Hirshfield, in Advanced Accelerator Concepts: Eighth Workshop, edited by W. Lawson, C. Bellamy, and D. Brosius, AIP Conf. Proc. No. 472 (AIP, New York, 1999), p. 27.

[2] J-M. Fang, T. C. Marshall, J. L. Hirshfield, M. A. LaPointe, T-B. Zhang, and X. J. Wang, in Proceedings of the Particle Accelerator Conference, New York, 1999 (IEEE, Piscataway, NJ, 1999), p. 3627.

[3] S. Y. Park and J. L. Hirshfield, Phys. Rev. E 62, 1266 (2000), and references therein.

[4] J. G. Power, W. Gai, and A. Kanareykin, in Advanced Accelerator Concepts: Ninth Workshop, edited by P.L. Colestock and S. Kelley, AIP Conf. Proc. Vol. 569 (AIP, New York, 2001), p. 605, and references therein.
[5] I. N. Onishchenko, D. Yu. Sidorenko, and G. V. Sotnikov, Phys. Rev. E 65, 066501 (2002), and references therein.

[6] L. Schächter, R. L. Byer, and R. H. Siemann, Phys. Rev. E 68, 036502 (2003), and references therein.

[7] L. Schächter, Phys. Rev. E 69, 049901(E) (2004).

[8] J. G. Power, M.E. Conde, W. Gai, R. Konecny, and P. Schoessow, Phys. Rev. ST Accel. Beams 3, 101302 (2000).

[9] S. Y. Park, C. Wang, and J. L. Hirshfield, in Advanced Accelerator Concepts: Tenth Workshop, edited by Christopher E. Clayton and Patrick Muggli, AIP Conf. Proc. Vol. 647 (AIP, New York, 2002), p. 527.

[10] C. Wang, J.L. Hirshfield, and T.C. Marshall, in Proceedings of the Particle Accelerator Conference, Portland, OR, 2003 (IEEE, Piscataway, NJ, 2003), Vol. 3, p. 1930, and references therein.

[11] L. Xiao, W. Gai, and X. Sun, Phys. Rev. E 65, 016505 (2002).

[12] C. Wang, J. L. Hirshfield, J.-M. Fang, and T. C. Marshall, Phys. Rev. ST Accel. Beams 7, 051301 (2004).

[13] S. V. Shchelkunov, T.C. Marshall, J.L. Hirshfield, and M. A. LaPointe, Phys. Rev. ST Accel. Beams 8, 062801 (2005).

[14] S. V. Shchelkunov, Ph.D. dissertation, Columbia University, 2005.

[15] X. J. Wang, X. Qiu, and I. Ben-Zvi, Phys. Rev. E 54, R3121 (1996).

[16] X.J. Wang, M. Babzien, K. Batchelor, I. Ben-Zvi, R. Malone, I. Pogorelsky, X. Qui, J. Sheehan, J. Skaritka, and T. Srinivasan-Rao, Nucl. Instrum. Methods Phys. Res., Sect. A 375, 82 (1996).

[17] J. Mollá, R. Moreno, and A. Ibarra, J. Appl. Phys. 80, 1028 (1996), and references therein.

[18] S. V. Shchelkunov, J-M. Fang, T. C. Marshall, and J. L. Hirshfield, in Proceedings of the 2003 Particle Accelerator Conference, edited by J. Chew, P. Lucas, and S. Webber (IEEE, Piscataway, NJ, 2003), Vol. 3, p. 1924. 